\title{
Raspberry Pi based Security Surveillance with Face Detection
}

\author{
Ms. Patil Sahyadri N ${ }^{1}$, Mr. Devrukhkar Akshay $\mathrm{S}^{2}$ \\ Student, CSE, Yashoda Technical Campus, Satara, India ${ }^{1,2}$
}

\begin{abstract}
The Raspberry Pi is a credit-card sized chip or mini computer is used in surveillance to the security of thefts. This system is crucial for the safety purpose of these days. In this paper focuses on only detect the face by using Opencv (Open Source Computer Vision) technique. ARS (Audience Response System) captures and identify the face of the theft and then ARS system notify the user. The process of notification is done by sending short message service (SMS) through Global System for Mobile communication (GSM) Module. The people i.e. human faces, are one of most common and specific objects that we try to trace in images. Face Detection the first step is analyzing the face of thefts then detect the person. In this paper we presented various methods of face detection, which are commonly used. These are various methods are Local Binary Pattern (LBP).
\end{abstract}

Keywords: Raspberry pi, GSM module, Opencv, Surveillance, Camera, Local Binary Pattern (LBP).

\section{INTRODUCTION}

Surveillance and Face Detection has become most important technique for security purpose of these days. Surveillance system is enabled by high end camera, video server, network switch, face detection. In this paper monitoring system must be active enough to notify the person.

Raspberry pi is credit card sized chip used in surveillance to secure of thefts and notify the user. It is complex used for find the thefts so we are use the best solution whose name is Closed Circuit Camera (CCTV) and Open Source Computer Vision (Opens) these are two technique are used.

Raspberry pi is a single credit card sized chip intellectual enough to capture the footage reducing the size and price it means low costly. Surveillance and Face detection using Raspberry pi involves Opens and Simple CV, gather of libraries and software that allows used to work images and video that come from camera and it detects the theft and notify the user through surveillance system

There are two main algorithm for Face detection first the HAAR and second one is LBP.IN this paper we are using the LBP (Local Binary Patterns) algorithm for face detection in Opencv (Open Source Computer Vision Library), this algorithm is easy to use compare with HAAR algorithm. LBP algorithm or cascade is easy to perform and detect the image fastly better than HAAR cascade or algorithm.so we are use the LBP algorithm.

Following comparison between the HAAR and LBP Cascade: haarcascade_frontalface_alt $2 . x m l$

Data file: muct.csv

$\mid$\begin{tabular}{l|l|} 
Hits $\mid$ Misses & False Detects \\
$3635 \mid$ & Multi-hit |
\end{tabular}

Time: $4 \mathrm{~m} 2.060 \mathrm{~s}$

VS

Cascade: lbpcascade_frontalface.xml

Data file: muct.csv

\begin{tabular}{l|l|l|}
$\mid$ Hits | Misses | & False Detects & Multi-hit \\
$|3569| 106 \mid$ & 77 & 3
\end{tabular}

Time: $1 \mathrm{~m} 12.511 \mathrm{~s}$

\section{RELATED WORK}

A. Raspberry Pi

The Raspberry $\mathrm{Pi}$ is a credit card-sized chip or minicomputer developed in the United Kingdom. There are various types like $\mathrm{A}, \mathrm{B}$ and $\mathrm{B}+$.it is advanced RISC machine processed lightweight computer minimized onto a single board. RISC (Reduced instruction set computing) is a microprocessor designed to perform a smaller number of types of computer instruction so it can operate at a higher speed. ARM (Advanced RISC machines). ARM processors are extensively used in consumer electronic devices like smartphones, tablets, multimedia players and other mobile devices, like wearable. This technology used on the board reduces cost, heat. The $\mathrm{B}+$ is the latest version of Raspberry pi and it is most widely used of these days, its runs on ARM11 processor with 512mb RAM operate at $700 \mathrm{MHz}$ frequency. As show in above fig of Raspberry pi B+ board, there are many slots of that board including SD card slot, HDMI Port, Audio/Video jack, 
IARJSET

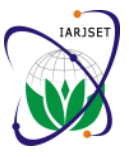

International Advanced Research Journal in Science, Engineering and Technology

National Conference on Innovative Applications and Research in Computer Science and Engineering (NCIARCSE-2017) AGTI's Dr. Daulatrao Aher College Engineering, Vidyanagar Extension, Karad

Vol. 4, Special Issue 4, January 2017

Ethernet, DSI Display Connector,40 pin JPIO pin headers,4 USB ports, etc.SD Card which can be use bootable device with Raspbian, pidora, Raspbmc etc. Raspberry pi most widely used Raspbian operating system.GPIO (general-purpose input/output) used in Raspberry pi.it is control Electronics devices like LEDs, Motor Driver, sensor so on using these GPIO pins.it is also used to connect devices to a computer so it can control and monitor them.

HDMI (High Definition Multimedia Interface) port is a digital interface, it can be connected to many devices like projector, PC, HDTV, LCD screen etc.HDMI extension switch connect several monitors or PC at a time.

Ethernet is the most widely installed on local area network (LAN).higher network protocol like internet protocol (IP) use Ethernet as their transmission medium. Ethernet OS most popular physical layer LAN technology in use today.

\section{B. GSM Module}

The GSM SIM900 is the module of global system for communication module, this module supports for communication in $900 \mathrm{MHz}$ band. First we are check the basic AT command run on global system for communication in raspberry pi then the commands sent via the serial port on Raspberry pi. The GSM modem with Sim900 module is make with Dual band GSM/GPRS. Baud rate can be configurable by using AT commands and it works on 900/1800 $\mathrm{MHz}$ frequencies. AT command operates on $12 \mathrm{v}$ regulated power supplies. GSM module is as shown in bellow figure it has one Sim card slot, one Antenna, one serial port and one power port. The SIM card slot to insert the SIM and an antenna to receive the network signals. The RS-232 is standard for serial communication of data.it is also used in computer serial ports. The RS-232 is connected to modems, printers, mice, data storage, power supplies and other peripheral devices. The GSM SIM900 module can perform the function on a mobile phone like sending the SMS and second receiving the SMS and TCP/IP communication over the GPRS on AT commands. In this paper the AT can send sms via Raspberry.

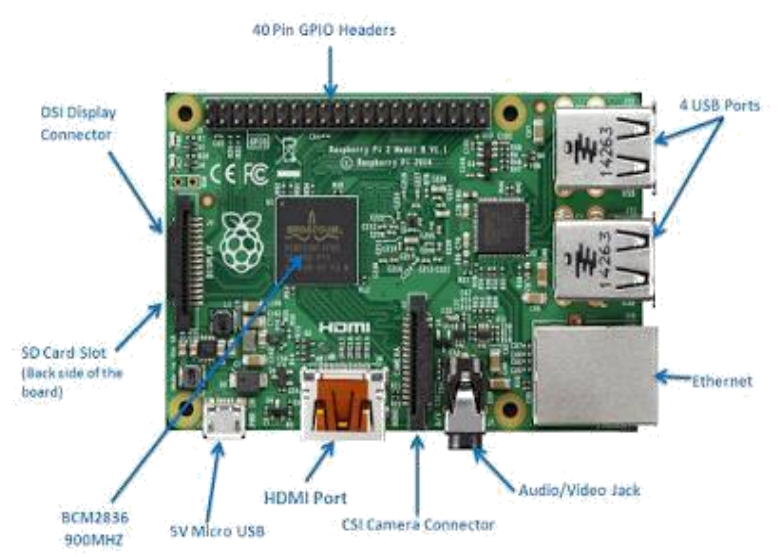

Figure 1 Raspberry Pi B+ Board

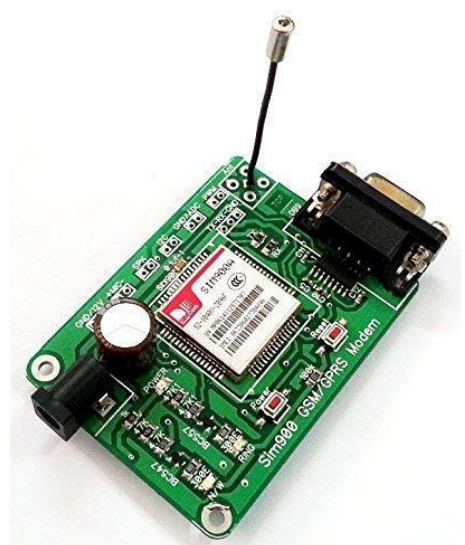

Figure 2 SIM900 GSM/GPRS Module

Global System for Mobile Communication (GSM) modem to control the home equipment's including light, conditional system and security system through short message service (SMS). The structure of this paper is as shown in bellow: Section III (A) explain the system design implementation of the surveillance system. Section III (B) explain the algorithm of the program whose name is coding algorithm for surveillance system.

\section{SimpleCV}

SimpleCV is the open source framework for computer vision application. The high power computer vision is the Opencv, without having to first learn many concepts including file formats, color, space, buffer management, bit depths. Simple CV is the interface of open source machine vision libraries in python. Use of simple $\mathrm{CV}$ template highlight text below through mouse and select the copy function from our browser then paste into your processor. Closed circuit television is also known as video Surveillance, It is most widely used video camera to transmit one signal to a specific place. Surveillance is powerful device and it is used by governments for intelligence gathering, the prevention of crime, the protection of process, person and it is also used for criminal organization, kidnapping etc. Surveillance is one type of the camera means the video camera and the main purpose of the video camera is observing an area and detect the thefts. It is covert observation of people, palace and vehicles and it is also known as private detective.

There are main two type of the surveillance is as show in bellow first is the tailing and second one is the shadowing. The security camera is the one point of the surveillance it provides the security for our home, workplace as well as providing with video footage of any events

\section{IMPLEMENTATION}

\section{A. System Design}

The System Design for surveillance system is as show in below figure, basic aim of this system design is to continuously capture the surveillance and if any government is noticed and the message send to the user notifying him is as show in above figure of system design. 
IARJSET

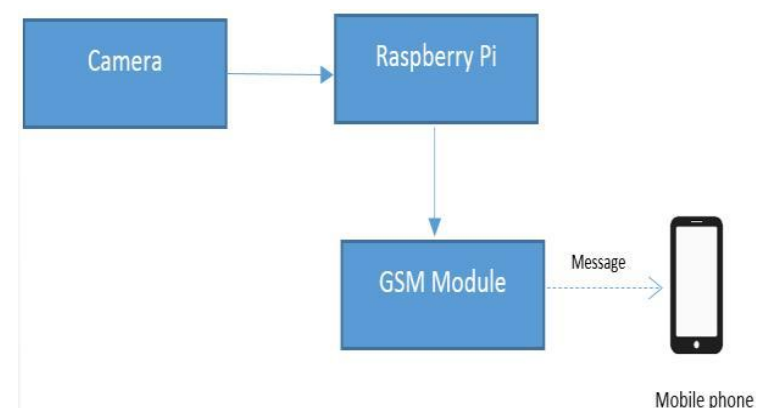

Figure 3 System design for surveillance system

The message send to user via GSM module short message service (SMS) it is connected to Raspberry pi through serial port of RS232.the program is written in programming language and capturing surroundings using camera and comparing them with the image frame.

System design is first capture the image then send the raspberry pi the raspberry pi process on that image and send to GSM module.

The Surveillance system uses MPEG streamer to stream live video on screen connected to Raspberry Pi via HDMI port and we can connect one or more computer or monitors at a time using HDMI (High-Definition Multimedia Interface) switch.

\section{B. Workflow Algorithm}

Raspberry Pi uses Raspbian operating system. Raspberry $\mathrm{Pi}$ supporting many programming language such as python, html5, JavaScript, c etc. The surveillance system python language is used because this language is easy to communicate with serial port, SimpleCV, Opencv modules.

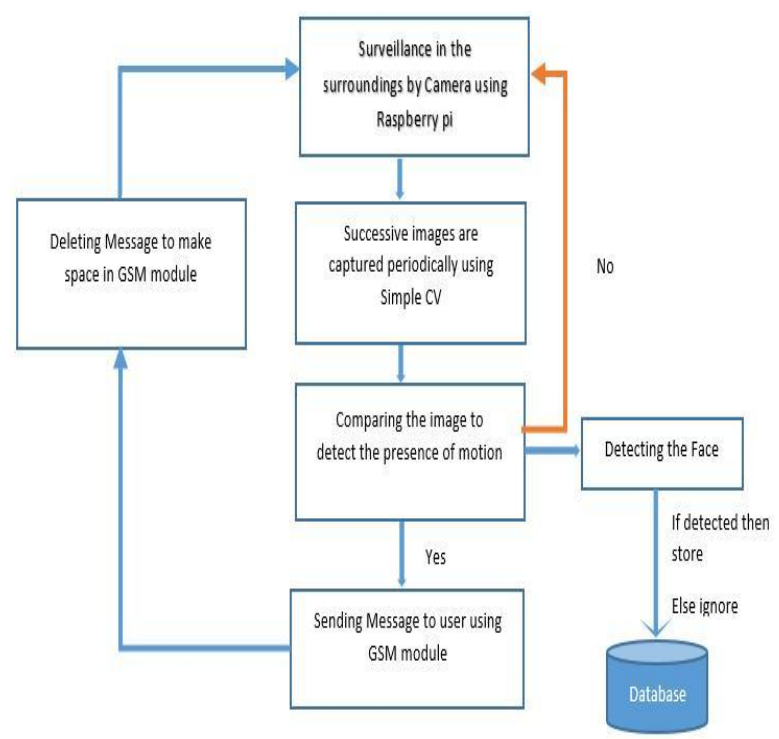

Figure 4 Workflow algorithm for surveillance system

The Raspberry Pi written program in python programming language using simple CV module the raspberry pi reads the data and capture the image by using simple CV then comparing the image to detect the Face and send the SMS (short message service) form GSM module via serial communication link. If detect the face then that data is store on database else ignore of that data and if not compare the image then send to the surveillance system of Raspberry Pi This system is as show in figure of workflow algorithm for Surveillance system.

The Surveillance system is implemented following step: Step 1. Serial communication and SimpleCV Module first import the required modules.

Step 2. Initialize the serial port for communicating to GSM module.

Step 3. The GSM module communicate with camera to read the image of face.

Step 4 . The image frame capture by using simple CV module.

Step 5. Consecutive of images are compare with each other.

Step 6. This comparing the image to detect the motion.

Step 7. If the notification is detect the send the notification to the user.

Step 8. IF the detection of the image is fail the surveillance system is continue.

Step 9. The notification is send to the user through the GSM (Global system for mobile) module, then the ARS system continues.

\section{CONCLUSION}

The proposed system is very efficient and much cost effective. The use of Raspberry Pi allows us to connect and program devices like camera, GSM module, displays etc. which increases its potential. The use of OpenCV and SimpleCV in this work makes image processing and surveillance more effective and easy to implement and manage. The face detection technique allows us to identify the thief or unknown person and it will become helpful in further investigation.

\section{REFERENCES}

[1] P. Vamsikrishna, S. Hussain, N. Ramu, P. Rao, "Advanced Raspberry Pi Surveillance (ARS) System" Proceedings of 2015 Global Conference on Communication Technologies (GCCT 2015)

[2] Sarthak Jain, Anant Vaibhav and Lovely Goyal, "Raspberry Pi based Interactive Home Automation System through E-mail", International Conference on Reliability, Optimization and Information Technology-ICROIT 2014, India, Feb6-8 2014.

[3] Nicholas D., Darrell B., Somsak S., "Home Automation using Cloud Network and Mobile Devices," IEEE Southeastcon 2012, Proceedings of IEEE. Laur, I., "Microcontroller based home automation system with security," International Journal of Advanced Computer Science and Applications, vol. 1, no. 6, pp. 60-65, 2010.

[4] T. parthornratt, N. Burapanonte, W. Gunjarueg, "People Identification and Counting System Using Raspberry Pi” IEEE coference, 2016

[5] J. Clerk Maxwell, A Treatise on Electricity and Magnetism, 3rd ed., vol. 2. Oxford: Clarendon, 1892, pp.68-73.

[6] Face Recognition with OpenCV - OpenCV 2.4.13.2 documentationhttp://docs.opencv.org/2.4/modules/contrib/doc/facer ec/facerec tutorial.html"

[7] SimpleCV official website [online] URL: http://simplecv.org/ 